\section{A cosmic history}

\author{
Millions of hours of processing time yield the \\ best picture so far of how the Universe evolved.
}

$\mathrm{I}$ $\mathrm{n}$ perhaps his most famous piece of scientific writing, the Italian chemist and Auschwitz survivor Primo Levi describes the story of a carbon atom. It begins its journey after being mined from a limestone prison that has sealed it for "hundreds of millions of years". Then, after more than a century of travels - including being inhaled by a falcon, turned into wine and locked up once again in the wood of a cedar tree - it finishes by fuelling the neural instruction that Levi's brain issues to scrawl the full stop that ends the story.

Included in his memoir The Periodic Table, the story of carbon, although brilliant and elegant, is fudged slightly. What happened to the carbon atom beforehand? Levi describes but a fraction of its lifespan. Indeed, he admits as much: "It already has a very long cosmic history behind it, but we shall ignore it."

'Cosmic history': even at their simplest, Levi's words have a lyricism that few can match. Carbon's turning up in a green leaf, he wrote, might "by full right deserve to be called a miracle". Most scientists interested in cosmic history these days call that carbon, and the leaf along with everything else we can hold, see, smell, touch or describe in stories of physical transformation - 'baryonic matter'.

Like Levi's single atom of carbon, the baryonic matter we see today, when we merely look out of our window or gaze at the majesty of the night sky, has a long cosmic history behind it. However, we can no longer ignore it. At least, not if we want to understand it - and how it has swirled and changed to form galaxies, stars, black holes and, yes, the odd leaf. Ironically, when physicists, mathematicians and cosmologists have tried to peer backwards into deep time, to model the way the Universe grew from its very beginnings, they have found it easier to focus on simulating the stuff that they understand the least: dark matter and dark energy.

On page 177 of this issue, scientists describe a computer simulation of baryonic matter that offers the clearest picture yet of its cosmic
"Watch as

13 billion years unwind and the matter of existence is forged from stardust." history - and also does a remarkable job of recreating in pixels the structure of the Universe we see around us. In strings of computer code and over some 16 million hours of processing time, giant gas clouds evolve and cool; stars form; supernovae explode and flood their region of space with energy; supermassive black holes churn; and, of course, chemical elements form, Levi's carbon atom somewhere among them.

As an accompanying News \& Views article on page 170 summarizes: "If all this sounds somewhat complicated, do not be fooled: it is extremely complicated." The results are not the final word, but they will allow researchers to test with data their ideas on, say, the physical processes that guide galaxy formation and the evolution of black holes.

The simulation also allows the scientists to turn the hard data into hypnotic videos that play out how the Universe grew and twisted into shape (see go.nature.com/rfjzy4). Peer back to a mere 12 million years after the Big Bang and then watch as 13 billion years unwind and the matter of existence is forged from stardust. Imagine among this an atom of carbon or a molecule of carbon dioxide, an "ever renewed impurity of the air", as Levi said, from which we all come. And then think where it could be now.

\section{The game is on}

\section{Amoeba races demonstrate a fun way to promote interest in science.}

$\mathrm{L}$ ater this month, an unusual scientific race will be run on the site of a former navy yard in Boston, Massachusetts.

The racetracks will be about 800 micrometres long, constructed from simple silicone mazes. Doping and genetic enhancement are not only allowed, but encouraged. And the racers will be Dictyostelium slime moulds, lured to the finish line by chemicals normally secreted by their favourite food - bacteria.

The trainers of the mould that wins on 16 May will get a US $\$ 5,000$ prize and 15 minutes in the spotlight at the Annual International Dictyostelium Conference in Potsdam, Germany, in August. For the victorious 'Dicty'? Perhaps a little extra sugar added to its culture medium, jokes bioengineer Daniel Irimia, who is hosting the competition in his laboratory at the Massachusetts General Hospital.

The Dicty World Race is a refreshing and beguiling way to bring attention to legitimate scientific questions: what guides cell migration and how can it be manipulated? The answers could inform our understanding of how cells move to the correct position in a developing embryo, or how immune-system cells flock towards invading bacteria. The race will also pit Dicty against human leukaemia cells, which are often faster than the slime mould, but are not as adept at navigating mazes.

The cash prize has been donated by members of the public and could be bigger by race day, but the winner is unlikely to be able to retire on the proceeds. Nevertheless, a prize was important, Irimia says, to lure researchers who do not normally study Dicty. Judging from the mix of 20 teams that have signed up for the race, the strategy has worked. Irimia hopes that the race will also introduce Dicty researchers to the power of rigorously quantifying their cell-migration assays, and to methods they can use to do so.

Since it first appeared in the scientific literature in 1935, Dictyostelium discoideum and its related species have built a surprisingly large and devoted fan base. The government mycologist who first described D. discoideum - having scraped it off decaying leaves in the mountains of North Carolina - prized it for the ease with which he was able to cultivate it on dung-agar plates. Later researchers would also value its streamlined, easy-to-analyse genome, and the way its single, independent cells can aggregate into multicellular structures.

At its best, the coterie of Dicty researchers - like many other small scientific communities that congregate around cherished model organisms - stands as a model for how science should be conducted. The group is tight-knit and collaborative. Researchers trade protocols, strains and even the occasional unpublished result. They sing the praises of their beloved Dicty, and generally prefer the respectful descriptor 'social amoeba' to the disparaging 'slime mould'.

Furthermore, the Dicty World Race is an example of an emerging way to stimulate scientific discussion. A similar spirit is evoked by computer games that draw on the power of crowds to solve protein structures (see Nature 466, 685-687; 2010) and trace neural connections. There are also scientific games based on microfluidic devices that allow players to manipulate living creatures, such as protozoa, using electric fields or chemicals. In 'the prisoner's smellemma' - a variation of the prisoner's dilemma strategy game - players mix samples of yeast or buffer in a test tube, and then smell the result to guess what their opponent used.

Such games are positive for science, and we should encourage their spread. An engaging way to attract attention not
DNATURE.COM To comment online, click on Editorials at: go.nature.com/xhunqu only from researchers in other fields, but also from the public, they highlight — and perhaps rejuvenate - the sense of play that drew many to science in the first place. 